\title{
Intususcepción yeyunogástrica retrógrada en un paciente con historia de gastroyeyunostomía hace 20 años: reporte de caso y revisión de la literatura
}

\section{Retrograde jejunogastric intussusception in a patient with underlying history of gastrojejunostomy 20 years ago: Case report and literature review}

\author{
Rolando Medina-Rojas, ${ }^{1}$ (1) Yesid Quintero-Pérez. ${ }^{*}$ (])
}

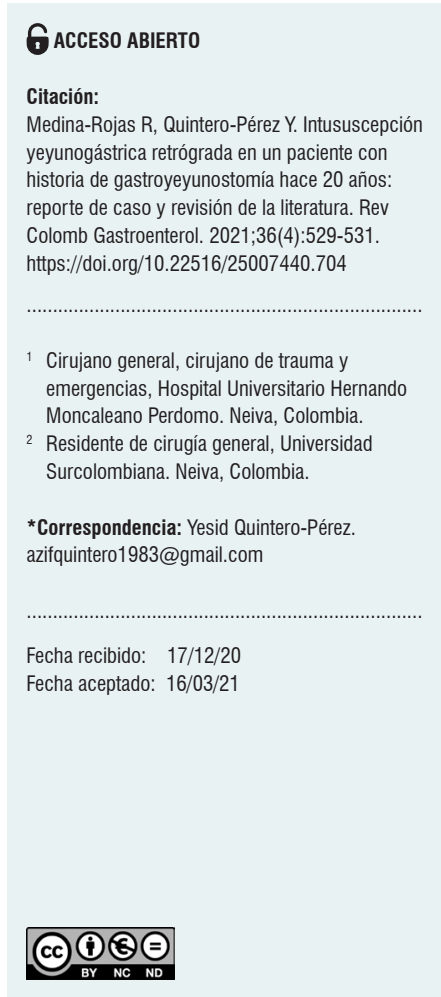

\begin{abstract}
Resumen
La intususcepción yeyunogástrica es una complicación rara, pero potencialmente fatal de acuerdo con el momento de su diagnóstico e intervención. Debido a su baja incidencia, se requiere de una alta sospecha diagnóstica, basada en la clínica y los antecedentes quirúrgicos. En el presente reporte se expone el caso de un paciente de 74 años, con historia de gastroyeyunostomía y cerclaje duodenal realizados como parte del manejo de úlcera duodenal 20 años atrás. Se presentó por urgencias luego de 7 días de inicio de los síntomas caracterizados por dolor, intolerancia a la vía oral y hematemesis. Su diagnóstico se realizó mediante endoscopia de vías digestivas altas y su manejo definitivo, mediante gastrectomía subtotal y reconstrucción en $Y$ de Roux transmesocólica por laparotomía.
\end{abstract}

\section{Palabras clave}

Intususcepción yeyunogástrica, complicación posoperatoria de gastrectomía, intususcepción yeyunogástrica retrógrada, invaginación, cirugía gástrica.

\begin{abstract}
Retrograde jejunogastric intussusception is a rare but potentially fatal complication, according to the time of diagnosis and intervention. Due to its low incidence, a high diagnostic suspicion is required, based on the clinical and surgical history. This study presents the case of a patient of 74 years old with a history of gastrojejunostomy and duodenal cerclage performed as part of duodenal ulcer treatment 20 years ago. The patient was admitted to the emergency unit, after 7 days of having symptoms such as pain, intolerance to oral intake, and hematemesis. Diagnosis was performed by upper digestive tract endoscopy and the final treatment by subtotal gastrectomy and transmesocolic Roux-en-Y reconstruction by laparotomy.
\end{abstract}

\section{Keywords}

Jejunogastric intussusception, Post operatory complication of gastrectomy, Retrograde jejunogastric intussusception, Invagination, Gastric surgery.

\section{INTRODUCCIÓN}

La intususcepción posoperatoria en adultos en cirugía gástrica tiene una incidencia menor del $0,1 \%$, por lo que es una complicación rara; y de estas, la más comúnmente reportada es la intususcepción yeyunogástrica. El primer caso reportado en la literatura se realizó en 1914 por Bozzy luego de una gastroyeyunostomía ${ }^{(1,2)}$.

El tiempo de diagnóstico y resolución es crucial para su pronóstico, ya que en los diferentes reportes de casos disponibles en la literatura se describen tasas de mortalidad que llegan hasta el $10 \%$ cuando el diagnóstico se realiza de 
forma temprana (antes de las 48 horas), pero esta misma mortalidad asciende hasta el $50 \%$ cuando el diagnóstico se realiza después de dicho tiempo ${ }^{(1,2)}$.

Solo el $50 \%$ de los pacientes se presenta con la tríada clásica de síntomas que incluyen dolor epigástrico y vómito con o sin hematemesis y masa palpable. Una vez hecho el diagnóstico, la realización de laparotomía para la reducción o resección de la intususcepción es el método de manejo más común. Recientemente se han documentado reportes de manejos endoscópicos y laparoscópicos exitosos ${ }^{(1-3)}$.

\section{REPORTE DE CASO}

Se trata de un hombre de 74 años con múltiples morbilidades de origen hipertensivo y cerebrovascular, con historia de 20 años de gastroyeyunostomía y cerclaje duodenal como parte del manejo quirúrgico por úlcera péptica perforada del antro gástrico. Refirió al ingreso un cuadro clínico de 7 días de evolución de dolor epigástrico, inapetencia y vómito posprandial ocasional manejado de forma empírica con antiácidos y remedios caseros, se presentó al departamento de urgencias por exacerbación aguda de los síntomas descritos de 8 horas de evolución, asociado con hematemesis fétida en tres oportunidades. En el examen físico se identificó dolor epigástrico, distensión y sensación de masa en el hipocondrio derecho. Se inició el manejo con inhibidores de la bomba de protones (IBP) en infusión y manejo médico sintomático. En los laboratorios de ingreso se encontró leucocitosis leve, hemoglobina normal $(13,9$ $\mathrm{g} / \mathrm{dl}$ ), función renal y tiempos de coagulación normales. Durante el curso de su estancia presentó nuevos episodios de hematemesis asociados con taquicardia, palidez mucocutánea marcada y compromiso del estado general, por lo que se envía a la unidad de cuidado crítico para vigilancia hemodinámica e inicio de soportes vasopresores.

Se realizó una endoscopia de vías digestivas altas en la que se encontró el asa yeyunal intususceptiva en la cámara gástrica de aspecto isquémico, por lo que se llevó a sala de cirugía para la realización de laparotomía exploratoria para gastrectomía subtotal y reconstrucción en Y de Roux transmesocólica. El paciente evolucionó de forma tórpida en el posoperatorio, por lo que requirió de dosis cada vez más altas de vasopresores duales y, finalmente, falleció pese a los manejos establecidos. El reporte del estudio anatomopatológico de la pieza quirúrgica reportó cambios isquémicos e inflamación aguda transmural.

\section{DISCUSIÓN}

La intususcepción es más común en la población pediátrica, solo el $5 \%$ ocurre en adultos, de los cuales se encuentra una causa desencadenante en el 70\%-90 \%; y de estas, la causa más común es lesiones malignas ${ }^{(4)}$. La intususcepción después de cirugía gástrica es una complicación rara, con una incidencia no mayor del 0,1\% y menos de 200 casos reportados ${ }^{(1,2,5)}$.

De acuerdo con la anatomía, existen tres tipos de intususcepción gastroyeyunal: en la tipo 1 , el asa aferente está intususceptiva en el estómago; en la tipo 2, el asa intususceptiva es la eferente y esta es la más comúnmente reportada (en el $80 \%$ de lo casos, como en este que describimos), y en la tipo 3, las asas aferente y eferente están intususceptivas. Se ha sugerido que la intususcepción gastroyeyunal tipo 2 sea la más común debido a una mayor morbilidad cuando el asa comprometida es la eferente frente a la aferente ${ }^{(2)}$ (Tabla 1).

Tabla 1. Clasificación anatómica

\begin{tabular}{|clc|}
\hline Tipo & \multicolumn{1}{c|}{ Asa yeyunal involucrada } & Frecuencia \\
\hline 1 & Asa aferente intususceptiva (anterógrada) & $16 \%$ \\
\hline 2 & Asa eferente intususceptiva (retrógrada) & $74 \%$ \\
\hline 3 & Forma combinada & $10 \%$ \\
\hline
\end{tabular}

Varias teorías fisiopatológicas funcionales y mecánicas se han postulado como desencadenantes de esta patología, pero ninguna ha podido confirmarse; dichas causas son antiperistalsis con emesis, existencia de adherencias peritoneales, dilatación intestinal posterior a la gastrectomía, hiperacidez, yeyunitis que causa peristalsis retrógrada, acortamiento del mesenterio del asa yeyunal y estenosis yeyunal con obstrucción que favorece la antiperistalsis ${ }^{(6)}$. Se han descrito tres tipos de presentación clínica: el tipo agudo usualmente ocurre con la tríada clínica de presentación súbita de dolor terebrante en el epigastrio, vómito con o sin hematemesis y masa palpable epigástrica; el tipo crónico recurrente se caracteriza por presentar dolor abdominal intermitente y llenura posprandial, en el cual los síntomas pueden resolverse de forma espontánea, y el tipo agudo posoperatorio, presente usualmente hacia el cuarto o quinto día posquirúrgico, se presenta de forma intermitente y se reduce espontáneamente ${ }^{(1)}$.

El diagnóstico oportuno determina el pronóstico, de modo que los pacientes diagnosticados antes de las 48 horas presentan tasas de mortalidad menores del $10 \%$ $y$, por el contrario, los que se diagnostican después de este tiempo presentan tasas de mortalidad del $50 \%^{(1,2,7)}$. Las herramientas de diagnóstico más importantes son la radiografía de abdomen, en la que ocasionalmente se puede encontrar masa en el cuadrante superior izquierdo, correspondiente con la intususcepción del intestino del- 
gado en el estómago; tomografía abdominal contrastada, y la endoscopia de vías digestivas altas, la cual permite la visualización directa del asa intususceptiva, la evaluación de la viabilidad de la misma y el diagnóstico de lesiones intraluminales asociadas. Se han descrito algunos reportes de reducción endoscópica de intususcepción con un riesgo elevado de recurrencia. La aplicación de estas técnicas de manejo endoscópico está proscrita en los casos en los que se sospecha irritación peritoneal ${ }^{(1,2,4)}$.
Ante el diagnóstico de intususcepción yeyunogástrica, el manejo médico tiene poco valor y la cirugía sigue siendo el tratamiento de elección. El tipo de procedimiento a realizar depende de los hallazgos endoscópicos e intraoperatorios de viabilidad intestinal, y se han descrito la resección, reducción del asa intususceptiva y la fijación de la misma a los tejidos adyacentes para disminuir el riesgo de recurrencia, como el estómago, el colon, el mesocolon ${ }^{(2-7)}$ y el ligamento falciforme ${ }^{(1)}$.

\section{REFERENCIAS}

1. Loi CM, Huang SY, Chen YD, Chen SD, Wu JM, Chen KH. Retrograde jejunogastric intussusception: A case report and review of the literature. Asian J Surg. 2017;40(4):309-312. https://doi.org/10.1016/j.asjsur.2014.04.001

2. Reyelt WP Jr, Anderson AA. Retrograde jejunogastric intussusception. Surg Gynecol Obstet. 1964;119:1305-11.

3. Archimandritis AJ, Hatzopoulos N, Hatzinikolaou P, Sougioultzis S, Kourtesas D, Papastratis G, Tzivras M. Jejunogastric intussusception presented with hematemesis: a case presentation and review of the literature. BMC Gastroenterol. 2001;1:1. https://doi.org/10.1186/1471-230x-1-1

4. Hui GC, Gerstle JT, Weinstein M, Connolly B. Small-bowel intussusception around a gastrojejunostomy tube resulting in ischemic necrosis of the intestine. Pediatr Radiol.
2004;34(11):916-8. https://doi.org/10.1007/s00247-004-1230-8

5. Ravirajendran S, Munnamgi S, Abdul A. Antegrade jejunojejunal intussusception inside a retrograde jejunogastric intussusception (double intussusception)-A rare case report. Int J Surg Case Rep. 2017;39:264-266. https://doi.org/10.1016/j.ijscr.2017.08.036

6. Tokue H, Tsushima Y, Arai Y, Endo K. Jejunogastric intussusception: life-threatening complication occuring 55 years after gastrojejunostomy. Intern Med. 2009;48(18):1657-60. https://doi.org/10.2169/internalmedicine.48.2115

7. Gopalakrishnan R, Ravikumar NG, Veerasamy M. Retrograde jejunogastric intussusception: A case report and review of literature. Arch Int Surg. 2014;4(1):47-9. https://doi.org/10.4103/2278-9596.136715 